\title{
Aromatic Hydrocarbons, Diamonds, and Fullerenes in Interstellar Space: Puzzles to be Solved by Laboratory and Theoretical Astrochemistry
}

\author{
K. Sellgren ${ }^{1}$ \\ Astronomy Department, Ohio State University, 140 West 18th Avenue, Columbus, OH \\ 43210 USA
}

\begin{abstract}
New research is presented, and previous research is reviewed, on the emission and absorption of interstellar aromatic hydrocarbons. Emission from aromatic hydrocarbons dominate the mid-infrared emission of many galaxies, including our own Milky Way galaxy. Only recently have aromatic hydrocarbons been observed in absorption in the interstellar medium, along lines of sight with high column densities of interstellar gas and dust. Much work on interstellar aromatics has been done, with astronomical observations and laboratory and theoretical astrochemistry. In many cases the predictions of laboratory and theoretical work are confirmed by astronomical observations, but in other cases clear discrepancies exist which provide problems to be solved by a combination of astronomical observations, laboratory studies, and theoretical studies. The emphasis of this paper will be on current outstanding puzzles concerning aromatic hydrocarbons which require further laboratory and theoretical astrochemistry to resolve. This paper will also touch on related topics where laboratory and theoretical astrochemistry studies are needed to explain astrophysical observations, such as a possible absorption feature due to interstellar "diamonds" and the search for fullerenes in space.
\end{abstract}

\footnotetext{
${ }^{1}$ Corresponding author: Tel.: 614-292-1898; fax: 614-292-2928; e-mail address: sellgren@astronomy.ohio-state.edu
} 


\section{Introduction}

Interstellar atomic gas, molecular gas, and dust grains, where grains are solid particles with sizes of $1-1000 \mathrm{~nm}$, pervade the space in between the stars known as the interstellar medium (ISM). The grains and molecules in the lower density regions of our Galaxy, called the diffuse ISM, are believed to form primarily in the outflowing gas from dying stars with masses similar to that of our own Sun. The final stage of this type of stellar death is a shell of ejected gas, known as a planetary nebula, which surrounds the stellar corpse, known as a white dwarf. Grains and molecules find it difficult to form in the general ISM, which has typical gas densities of $1-10$ atoms $\mathrm{cm}^{-3}$, and thus extremely low chemical reaction rates.

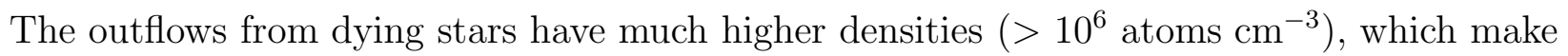
chemical reactions leading to formation of molecules and grains much more probable.

In our universe, the pattern of cosmic elemental abundances is determined first by the hydrogen and helium formed in the Big Bang, when our universe began, and secondly by nuclear reactions within stars, which account for virtually all the other elements. As a result of the pattern of common nuclear reactions within stars, only a very restricted set of elements are important in interstellar chemistry. Figure 1 shows the cosmic abundance, relative to hydrogen, of all elements which have an abundance larger than $10^{-6}$ relative to hydrogen [1]. It can be seen that the most important elements for interstellar chemistry are H, O, C, N, Mg, Si, Fe, S, Al, Ca, Na, and Ni. The inert gases He, Ne, and Ar are also relatively abundant but do not interact chemically. Other elements exist in the universe, but their scarcity leads to their playing a minor role in interstellar chemistry. By far the most abundant interactive elements are H, O, C, and N, and thus it is no surprise that the overwhelming majority of interstellar molecules detected to date are primarily composed of these elements.

The interstellar infrared emission features (IEFs), at 3.3, 6.2, 7.7, 8.6, 11.3, and 12.7 $\mu \mathrm{m}\left(790,890,1160,1300,1610\right.$, and $\left.3040 \mathrm{~cm}^{-1}\right)$, dominate the mid-infrared emission of our own and other galaxies. Figure 2 shows the $600-2000 \mathrm{~cm}^{-1}$ spectrum of one region within our own Galaxy, NGC 7023 [2]. This figure shows most of the IEFs. Note that this figure is plotted versus $\mathrm{cm}^{-1}$, to be helpful to chemists, but that the astronomical convention is to plot spectra versus $\mu \mathrm{m}$ and that the astronomical convention will be followed for the remainder of this paper.

The IEFs, which have widths broader than natural broadening mechanisms for lines in atomic gas or simple molecular gas, were mainly first discovered by astronomical observations in the 1970's [3 - 6], with the $12.7 \mu \mathrm{m}$ IEF discovered only after higher spectral resolution observations [7] were able to distinguish it from $\mathrm{Ne}^{+}$emission at 12.8 
$\mu \mathrm{m}\left(780 \mathrm{~cm}^{-1}\right)$. The IEFs have been referred to by a variety of names in the literature, with "feature" and "band" used interchangeably: the "unidentified emission features," the "unidentified infrared bands," the "infrared emission features," the "aromatic infrared bands," and the "polycyclic aromatic hydrocarbon (PAH) bands." The origin of the last two names will become apparent in later paragraphs.

The IEFs are believed to arise in either interstellar grains or interstellar molecules, and because their emission can account for up to $30-40 \%$ of the overall emission of galaxies, solving problems related to their composition, size, and origin are essential for understanding the overall energy budget of radiation from galaxies. Within our galaxy, the IEFs are observed in a wide variety of environments. One important clue to the composition of the carrier of the IEFs, however, is the astronomical observation that the strength of the $7.7 \mu \mathrm{m}$ IEF shows a strong correlation with the ratio of carbon to oxygen in planetary nebulae $[8-10]$. Ordinarily there is more oxygen than carbon in interstellar gas and in stars, which results in an interstellar chemistry in which common elements most often combine with hydrogen and oxygen to form molecules and grains. The heavier elements in such an oxygen-rich environment generally condense into solid dust grains composed of oxides of these elements, such as silicate grains which are commonly observed in the ISM. In some dying stars, however, convection within the star dredges up so much carbon, produced in nuclear reactions at the star's center, that the composition of the outflowing gas from a dying star changes to a peculiar situation in which the gas has more carbon than oxygen. This carbon-rich gas alters the chemistry of grain and molecule formation in the outflowing gas, preferentially forming molecules and grains which are rich in hydrogen and carbon. The observed correlation between the $7.7 \mu \mathrm{m}$ IEF strength and the $\mathrm{C} / \mathrm{O}$ ratio in planetary nebulae strongly suggests that the IEFs form from dust and/or molecules condensed in the outflows from dying stars with carbon-rich outflows. Once the IEFs, like other grains and molecules, are formed, the gas ejected from the dying star slowly mixes with the surrounding gas and dust within the galaxy and becomes part of the general ISM. The ISM in galaxies, therefore, contains a mixture of molecules and grains formed in oxygen-rich environments and carbon-rich environments.

Astronomical observations show strong emission from the IEFs in many regions of star formation, where dust and gas are interacting with the ultraviolet (UV) and visible radiation emanating from young stars or from stars in the process of forming (protostars). Two examples of star formation regions with strong IEF emission are reflection nebulae, where grains within a cloud of gas and dust scatters the light of a nearby young star, and photodissociation regions surrounding H II regions (see reviews by $[11,12]$ ). An H II region is the astronomical name for a cloud of ionized hydrogen gas surrounding a young star hot enough to ionize hydrogen; a photodissociation region is the astronomical name for the 
surface of a cloud of molecular hydrogen gas, where the UV radiation from a hot star is in the process of photodissociating the molecular hydrogen. Regions of the ISM are commonly referred to by the state of their hydrogen gas - neutral (H I), ionized (H II), or molecular $\left(\mathrm{H}_{2}\right)$ - because $71 \%$ of the mass of interstellar gas is composed of hydrogen. The hydrogen gas in reflection nebulae can be either neutral or molecular, but not ionized.

Astronomical mapping and images of the IEFs in the $\mathrm{H}$ II regions and adjacent photodissociation regions in the star-forming Orion Nebula and the planetary nebula NGC 7027 suggest that the IEFs are concentrated in a narrow zone between the ionization front and the photodissociation front $[13,14]$. The ionization front is where ionized hydrogen abruptly changes to neutral hydrogen at a distance from the ionizing star where all of the ionizing photons from the star has been absorbed by intervening hydrogen gas and re-radiated as several lower energy, non-ionizing photons. The ionization front can be delineated by astronomical observations of recombination lines of neutral hydrogen; such lines are strong in ionized hydrogen but absent from neutral hydrogen. The photodissociation front can be traced by astronomical observations of $\mathrm{H}_{2}$ emission; the same UV photons which can dissociate $\mathrm{H}_{2}$ have an alternate path in which they cascade through the electronic levels of $\mathrm{H}_{2}$ to populate vibrational-rotational levels of $\mathrm{H}_{2}$, which are then observed as $\mathrm{H}_{2}$ emission lines in the near-infrared $\left(1-2.5 \mu \mathrm{m}\right.$ or $\left.4000-10,000 \mathrm{~cm}^{-1}\right)$.

The IEFs are also observed in regions of low UV intensity, such as the surfaces of molecular clouds and the general ISM where the illumination source is the diffuse interstellar radiation field [15 - 17]. The ubiquity of IEF emission within our Galaxy, within star formation regions, within carbon-rich planetary nebulae, and within the general ISM, makes understanding their carriers an essential part of understanding how our Galaxy emits, absorbs, and reprocesses radiation. The prevalence of IEF emission in star formation regions, and the recent detection of IEF absorption toward protostars [18 - 23], also illuminates the important role that the IEF carriers play in the material from which stars and planets form.

The IEFs were first proposed to be due to aromatic hydrocarbons by Duley \& Williams [24], based on wavelength coincidences between the IEFs and laboratory spectra of aromatic hydrocarbons. Another clue to the composition of the IEF emitters was contributed by Sellgren et al. [25] and Sellgren [26], when we discovered $1-5 \mu \mathrm{m}\left(2000-10,000 \mathrm{~cm}^{-1}\right)$ continuum emission in reflection nebula that was not due to scattered starlight. Starlight which is scattered from grains is strongly polarized, and this infrared continuum emission in reflection nebulae was unpolarized [27]. This continuum emission had a color temperature around $1000 \mathrm{~K}$, and was spatially associated with IEF emission in these sources. The color temperature of the continuum emission showed no decrease with distance from the exciting 
star, as would be expected for thermal emission by dust in equilibrium with the star's radiation field. Furthermore the stellar radiation field was far too weak to heat grains to equilibrium temperatures of $1000 \mathrm{~K}$ at the observed nebular distances. Temperatures of $\sim$ $30-60 \mathrm{~K}$ were expected instead, and in fact dust in this temperature range had already been observed [28, 29] at far infrared wavelengths $\left(40-400 \mu \mathrm{m}\right.$ or $\left.25-250 \mathrm{~cm}^{-1}\right)$. We proposed a new paradigm for interstellar dust, in which the dust size distribution contained a component of $1 \mathrm{~nm}$ sized grains which were so small that a single UV photon absorbed from a nearby star could briefly heat the grain to temperatures as high as $1000 \mathrm{~K}$. Tiny particles of this size has been proposed earlier [30, 31]. These tiny grains spend the vast majority of their time at low temperature, but when they undergo stochastic heating they can for a microsecond or millisecond radiate emission at high temperatures [25, 26]. This model explains the high color temperature of the continuum emission, the constancy of the color temperature with distance from the exciting star, and the lack of polarization. Once we had proposed this model, several groups quickly realized the importance of this emission mechanism to the newly emerging data from the Infrared Astronomical Satellite (IRAS), launched in 1984, because the tiny grains, as they cooled radiatively, would be characterized by progressively lower color temperatures with time and thus contribute significantly to emission in the IRAS 12 and $25 \mu \mathrm{m}\left(400\right.$ and $\left.830 \mathrm{~cm}^{-1}\right)$ broad-band photometric filters [32 $-34]$.

The real breakthrough in understanding the IEFs, however, was made by Léger \& Puget [35] and Allamandola et al. [36]. They were the first to combine the aromatic hydrocarbon idea [24] with a $1 \mathrm{~nm}$ particle size [26], and to propose that the IEFs were due to polycyclic aromatic hydrocarbon (PAH) molecules with a size of roughly $1 \mathrm{~nm}$ or smaller. Other aromatic materials have been proposed for the IEFs, including tiny grains composed of hydrogenated amorphous carbon, quenched carbonaceous composite, and coal-like materials [37 - 42]. Virtually all astronomers working on the IEFs agree on the aromatic nature of the IEFs. There is still debate over the exact nature of the interstellar aromatics, but many favor the PAH hypothesis because it so neatly ties together well-studied aromatic materials with the $1 \mathrm{~nm}$ sizes suggested by the continuum emission associated with the IEFs.

The goal of the astronomical observational research of myself and my collaborators has been to test specific predictions of the PAH hypothesis, and to urge chemical theorists and laboratory astrochemists to make modifications in the PAH hypothesis when the predictions and observations do not match. This interplay between the observations, theory, and laboratory work has proven very fruitful in refining our understanding of the size, composition, ionization state, and hydrogenation of the material responsible for the IEFs in the ISM. 


\subsection{A Challenge: Approximating Interstellar Conditions in the Laboratory}

One of the most difficult challenges in matching astronomical observations and laboratory measurements of candidate materials for the IEFs is the vast gulf between interstellar conditions and laboratory conditions. The densities in interstellar space, even in the densest interstellar clouds, are so low that they exceed the capabilities of the best vacuum systems on Earth. The vast majority of published laboratory work has been measurements of solid-phase, room temperature aromatic materials in absorption, which are then compared to astrophysical observations of $\sim 1000 \mathrm{~K}$ aromatic materials in emission in extremely low density environments. Some progress has been made in approximating the low densities of interstellar conditions by use of inert-gas matrix isolation techniques, but even in these cases matrix interactions introduce frequency shifts in the wavelengths of absorption lines of unknown magnitude and direction. Other progress has been made in measuring aromatic molecules in emission in the laboratory, which has helped to quantify the temperature dependence of wavelength shifts in PAHs. Recent evidence, discussed

below, suggests that the PAHs that have been best-studied in the laboratory are too small to approximate interstellar PAHs. Furthermore, astrophysical theoretical calculations predict that PAHs should be ionized in the best-observed regions of the ISM. Both chemical quantum calculations and laboratory measurements demonstrate marked changes in the relative intensities and central wavelengths of PAH bands with ionization state. All of these mismatches between interstellar conditions and laboratory conditions make it difficult to achieve a firm identification of the material(s) responsible for the IEFs.

\section{The IEFs and the Hardness of the UV Radiation Field}

One of the early predictions of the PAH model is that PAH molecules should only be excited by UV radiation, since laboratory absorption curves of small, neutral PAHs show a sharp cutoff in their absorption at UV wavelengths with little or no absorption at visible wavelengths. Sellgren et al. [43] tested this prediction by using observations from the IRAS satellite to search for infrared emission in reflection nebulae excited by stars of different effective temperature, $T_{\text {eff }}$. We first verified that each reflection nebula was indeed excited by the central star of the nebula, by requiring that the dust temperature derived from the the ratio of $60 \mu \mathrm{m}\left(170 \mathrm{~cm}^{-1}\right)$ intensity to $100 \mu \mathrm{m}\left(100 \mathrm{~cm}^{-1}\right)$ intensity reach a peak value at the star. The emission at 60 and $100 \mu \mathrm{m}$ is thought to primarily come from grains, with a size around $100 \mathrm{~nm}$, which are in equilibrium with the radiation field, and whose 
temperature therefore will increase with proximity to the heating star. We then examined the $12 \mu \mathrm{m}$ emission of each reflection nebula, at a nebular position offset from the star so that stellar emission would not contaminate the $12 \mu \mathrm{m}$ data, and compared it to the total infrared emission at the same spatial location. For each nebula we then measured $R(12 /$ total $)$, the ratio of the total flux in the IRAS $12 \mu \mathrm{m}$ band to the total infrared flux. The IRAS $12 \mu \mathrm{m}$ band we assumed to be dominated by IEF emission and its associated continuum emission, as had been shown by mid-infrared spectroscopy for some of the sources in our sample [34]. We were expecting $R(12 /$ total) to show a precipitous drop for cool stars $\left(T_{\text {eff }}<10,000 \mathrm{~K}\right)$, where the fraction of total stellar radiation radiated in the UV was small. Much to our surprise, the value of $R(12 /$ total $)$ was independent of $T_{\text {eff }}$, over the range $T_{\text {eff }}=5,000 \mathrm{~K}-22,000 \mathrm{~K}$, as illustrated in Figure 3. Our observational results required that the material responsible for emitting the IRAS $12 \mu \mathrm{m}$ emission in reflection nebulae absorb not only at UV but also at visible wavelengths. This discovery altered the astronomical community's perception of PAHs as being small in size and neutrally charged, and drove theorists and laboratory astrochemists to consider both larger PAHs and ionized PAHs in their models and laboratory work. This is because increasing the PAH size and ionizing PAHs both have the effect of extending the absorption cross-section of PAHs out to visible wavelengths.

The $12 \mu \mathrm{m}$ IRAS filter bandpass is wide and encompasses several IEFs as well as their associated continuum. The material responsible for the continuum has never been identified, and the possibility exists that it is not due to the same material that produces the IEFs. In this case, it might be possible that the IRAS $12 \mu \mathrm{m}$ emission we observed in reflection nebulae illuminated by cool stars is due to continuum emission alone without any contribution from the IEFs. Thus, when the Infrared Space Observatory (ISO) was launched in 1995, we embarked on an observational program to obtain spectra of some of the reflection nebulae studied by Sellgren et al. [43], particularly reflection nebulae illuminated by both cool and hot stars which had similar values of $R$ (12/total) . These observations have been made with ISO's mid-infrared camera (ISOCAM) combined with its circular variable filter $(\mathrm{CVF})$, which allow us to obtain low-resolution $(\lambda / \Delta \lambda=40)$ spectra, at $5-15 \mu \mathrm{m}\left(600-2000 \mathrm{~cm}^{-1}\right)$, simultaneously at roughly a thousand spatial positions across each nebula. The ISO spectra of these reflection nebulae, therefore, should unambiguously determine whether the IEFs are present in these sources.

The first ISO results from Uchida et al. [44] was our discovery that IEFs are clearly detected in vdB 133, a reflection nebula illuminated by a binary system with very little UV radiation. The binary system, a luminous star with $T_{\text {eff }}=6,800 \mathrm{~K}$ plus a fainter star with $T_{\text {eff }}=12,000 \mathrm{~K}$, provides a ratio of UV $(\lambda<400 \mathrm{~nm})$ to total stellar flux which is a factor of four lower than more typical reflection nebulae which are illuminated by stars with 
$T_{\text {eff }} \sim 20,000 \mathrm{~K}$. Yet, despite the softer radiation environment, the IEF spectrum in this UV-poor environment is very similar to IEF spectra observed in sources with much harsher UV environments, as shown in Figure 4.

\subsection{New Results: Laboratory Analogs and the IRAS Data on R(12/total)}

I am currently collaborating with J. Pizagno, K. Uchida, and M. Werner on a comparison of various laboratory and theoretical candidates for the IEF carriers with the IRAS observations [43] of $R(12 /$ total), to quantify which materials can reproduce the lack of dependence of $R\left(12 /\right.$ total) on $T_{\text {eff }}$ (Pizagno et al. 2000, in preparation). We are convolving the UV and visible laboratory and theoretical absorption curves of different materials with the energy distributions emitted by stars of different $T_{\text {eff }}$, and comparing the results to our IRAS observations. Figure 3 shows one sample result of our calculations, where we compare the astronomical observations to the predicted results for two small PAH molecules, neutral naphthalene [45] and singly ionized naphthalene (from F. Salama 1999, private communication). These new results show that small neutral PAHs are completely unable to provide enough visible absorption to explain the IEF emission observed around UV-poor sources. They also show that while ionized PAHs have more visible absorption than their neutral counterparts, small PAHs even when ionized also cannot explain the IRAS observations.

\subsection{Need for Data and Theory for Larger PAHs and Varying Ionization}

It seems likely that a combination of ionization and larger PAH size will be required to explain the astronomical observations. We strongly encourage laboratory astrochemists to

measure parameters for larger PAHs, both neutral and ionized. We also strongly encourage theoretical astrochemists to calculate parameters for larger PAHs, both neutral and ionized, particularly for PAH sizes and/or ionization states that are not currently accessible by laboratory techniques.

\section{PAH Ionization}


Uchida et al. [46] have just completed a more comprehensive ISO study of IEFs in reflection nebulae, in which we have made unexpected observations which have important ramifications for models of PAH ionization. PAH ionization models predict that PAHs should be primarily positively charged in regions of high UV radiation, such as reflection nebulae, and primarily neutral or negatively charged in regions of low UV radiation, such as the diffuse ISM and cirrus clouds [47 - 50]. The UV radiation intensity is generally characterized by the variable $G_{0}$, where $G_{0}=1$ corresponds to the UV radiation field in the solar neighborhood. Laboratory and theoretical studies show that the ratio of PAH emission at $6-10 \mu \mathrm{m}\left(1000-1700 \mathrm{~cm}^{-1}\right)$ to the PAH emission at $10-14 \mu \mathrm{m}(700-1000$ $\mathrm{cm}^{-1}$ ) is a sensitive function of PAH ionization state, with this ratio varying by at least a factor of 10 between ionized and neutral PAHs [51 - 59].

We have measured the ratio of IEF emission at $6-10 \mu \mathrm{m}$ to the IEF emission at $10-14 \mu \mathrm{m}$, observed in reflection nebulae with $G_{0}$ varying from 20 to $6 \times 10^{4}$. Figure 5 shows that we see little or no evidence for any change in this ratio with $G_{0}$ (and thus PAH ionization state). This provides a major puzzle for the PAH hypothesis.

\subsection{PAH Ionization: Astrochemical Theory and Laboratory Data Needed}

There are several ways to resolve the discrepancy between the observations and theory for the predicted dependence of the PAH ionization state on the UV field. The calculated ionization states of PAHs are somewhat sensitive to the assumed PAH size. The amount by which the ratio of fluxes at $6-10 \mu \mathrm{m}$ to $10-14 \mu \mathrm{m}$ changes also depends on PAH size, with smaller changes in the ratio for larger PAHs. The calculated ionization states of PAHs are also very sensitive to the uncertain PAH recombination rate, which requires better laboratory measurements and/or better theoretical predictions for the recombination

rates, particularly as a function of PAH size. These are all areas in which astrochemistry in the laboratory and theoretical calculations can really make a difference in interpreting the astronomical observations.

\section{The Full-Width at Half Maximum of the $7.7 \mu \mathrm{m} \mathrm{IEF}$}

Another unexpected discovery Uchida et al. [46] have made with our recent ISO observations concerns spectral changes in the IEFs at low levels of illumination. We have 
quantitatively compared the IEF spectra (Fig. 4) of three sources we observed ourselves, vdB 133, vdB 17 (NGC 1333), and vdB 59 (NGC 2068), to the IEF spectra of published spectra of NGC 7023 ([2]; Fig. 1) and $\rho$ Oph [15]. We have found no evidence for any systematic spectral differences with $T_{\text {eff }}$. Our new ISO observations, however, find that the full width at half maximum (FWHM) of the $7.7 \mu \mathrm{m}$ IEF is dependent on the distance between star and nebula in vdB 17 [46]. Figures 6 and 7 show that in the most distant regions of $\mathrm{vdB} 17$, corresponding to the lowest UV illumination levels, $G_{0}=20-60$, the $7.7 \mu \mathrm{m}$ IEF becomes significantly broader and begins to blend with the $8.6 \mu \mathrm{m}$ IEF. This effect has now been observed in a second reflection nebula, Ced 201 [60], although they do not give the $G_{0}$ values at which the broadening of the $7.7 \mu \mathrm{m}$ IEF becomes significant. None of the other IEFs appear to change width with stellar distance in either source. We also find no broadening of the $7.7 \mu \mathrm{m}$ IEF or other IEFs in vdB 59, down to $G_{0}=200$. More significantly, the spectrum of $\rho$ Oph [15] at $G_{0}=40$ shows no sign of a broader 7.7 $\mu \mathrm{m}$ IEF or any blending of the $7.7 \mu \mathrm{m}$ IEF with the $8.6 \mu \mathrm{m}$.

The unexpected broadening of the $7.7 \mu \mathrm{m}$ IEF at low UV illumination levels, in some sources but not others, is very much a mystery. One possibility is that the intermittent broadening of the $7.7 \mu \mathrm{m}$ feature depends on two parameters, one being $G_{0}$, for instance, and the other being some factor such as $T_{\text {eff }}$. Stellar $T_{\text {eff }}$ seems like a good starting point for investigation, because vdB 17 and Ced 201, which are illuminated by stars with $T_{\text {eff }}=$ $10,000-11,000 \mathrm{~K}$, show the broadening of the $7.7 \mu \mathrm{m}$ IEF at low $G_{0}$ values, while $\rho$ Oph, which shows no such broadening at $G_{0}=40$, is illuminated by a pair of stars with $T_{\text {eff }}=$ $22,000 \mathrm{~K}$.

Two other ideas seem like promising avenues to explore. One is the recent ISO observations of Moutou et al. [61], illustrated in Figure 8. We find that the $7.7 \mu \mathrm{m} \mathrm{IEF}$, when observed at higher spectral resolution $(\lambda / \Delta \lambda=1800)$, appears to break up into at least three different features [61], at 7.45, 7.6, and $7.8 \mu \mathrm{m}\left(1280,1320\right.$, and $\left.1340 \mathrm{~cm}^{-1}\right)$. Previous astronomical spectra at $\lambda / \Delta \lambda=100-200[62,63]$ resolved the $7.7 \mu \mathrm{m}$ IEF into two features at 7.6 and $7.8 \mu \mathrm{m}$. Other astronomical observations [9, 10] at a lower resolution of $\lambda / \Delta \lambda=50$, where only the central wavelength of the $7.7 \mu \mathrm{m}$ IEF could be measured, demonstrated that the $7.7 \mu \mathrm{m}$ IEF central wavelength depends on the physical conditions in the emitting region (whether or not the hydrogen gas is ionized, for instance). Since the results of $[9,10]$ almost surely result from subtle changes in the relative strengths of the subcomponents of the $7.7 \mu \mathrm{m}$ IEF, then the broadening of the $7.7 \mu \mathrm{m}$ IEF observed at low resolution [46] could reflect more obvious changes in the relative strengths of the different subcomponents observed at higher resolution [61 - 63].

The other idea deserving further exploration is related to the fundamental reason why 
the IEFs are so much broader than gas molecules or grains moving at the Doppler speeds (1 $\mathrm{km} \mathrm{s}^{-1}$ or less) typical of these interstellar regions. There is no clear consensus on whether the IEF widths are due to physical processes (such as timescales for internal molecular processes, which depend on the size and temperature of the molecule) or to mixtures of aromatics with slightly different compositions, sizes, and central wavelengths. Observations of the IEFs in absorption suggest that the mixture of IEF carriers may be dominant in determining the central wavelength and width of the IEF emission, as discussed in the next section, and if this is the case, then the astronomical observations of changes in the central wavelength or FWHM of the $7.7 \mu \mathrm{m}$ IEF could reflect changes in the size distribution or composition of interstellar aromatics under different physical conditions.

\subsection{The 7.7 Micron IEF Width: Need for Laboratory and Theoretical Work}

Astrochemical work is desperately needed to formulate some theoretical framework for why the $7.7 \mu \mathrm{m}$ width changes, so that astronomical observations can be made to test this framework. As described above, the intermittent broadening of the $7.7 \mu \mathrm{m}$ IEF could depend on two parameters, one being $G_{0}$, for instance, and the other being some factor such as stellar temperature. Can laboratory or theoretical work provide any insights into why these two parameters, or some other set of parameters, might affect the $7.7 \mu \mathrm{m}$ width? Does the broadening of the $7.7 \mu \mathrm{m}$ IEF observed at low resolution reflect changes in the relative strengths of the different subcomponents at $7.45,7.6$, and $7.8 \mu \mathrm{m}$ observed at higher resolution [61]? Or do the relative strengths of the different subcomponents of the $7.7 \mu \mathrm{m}$ IEF stay the same, but the width of each subcomponent change? What physical change would be needed in the IEF carriers for the width to change? Could it be that the size distribution or composition of interstellar aromatics change with different physical conditions? Or is it some other phenomenon entirely? The answer to the questions posed by observations of the $7.7 \mu \mathrm{m}$ IEF are rooted in the ongoing debate as to the origin of the IEF widths themselves.

\section{Aromatics in Absorption}

Until recently, all astronomical observations of aromatic hydrocarbons in the ISM were of the IEFs in emission. The absorption signatures of the IEFs had not been seen. We 
have begun a program to try to trace the abundance and chemical changes of aromatic hydrocarbons during their residence time in molecular clouds. A broad $3.25 \mu \mathrm{m}(3080$ $\mathrm{cm}^{-1}$ ) absorption feature along the line-of-sight to the protostar Mon R2/IRS-3 was tentatively detected, and its discovery confirmed with better spectra, by Sellgren et al. [18, 19]. We proposed that this feature is due to the $\mathrm{C}-\mathrm{H}$ stretch absorption of cold aromatic hydrocarbons within the molecular cloud. Laboratory work on PAHs [64] has shown that the central wavelength of PAH features shifts to increasing wavelength with increasing temperature. This predicts that aromatics in absorption should be blue-shifted by about the observed amount, relative to aromatics in emission, because aromatics in emission are emitting at temperatures of $\sim 1000 \mathrm{~K}$, while aromatics in absorption are absorbing at much lower temperatures, $\sim 10-80 \mathrm{~K}$. In Brooke et al. [21, 22] we have now detected the 3.25 $\mu \mathrm{m}$ absorption feature toward a total of five protostars embedded in molecular clouds.

\subsection{New Results: Comparing Aromatics in Emission and Absorption}

The current observations of aromatics in absorption, compared to aromatics in emission, at first glance seem to have a simple interpretation. But there is an interesting complication. Ground-based [65] and ISO [66] spectra of sources near the Galactic Center reveal a narrow absorption feature near $3.28 \mu \mathrm{m}\left(3050 \mathrm{~cm}^{-1}\right)$, which these authors identify with aromatic hydrocarbons. If this feature is due to the $3.29 \mu \mathrm{m}\left(3040 \mathrm{~cm}^{-1}\right)$ IEF emitter, observed in absorption through the diffuse ISM in our Galaxy, it should spend the vast majority of its time at cold temperatures and thus have a wavelength blue-shifted relative to the $3.29 \mu \mathrm{m}$ IEF in emission, similar to the blue-shifted wavelength $(3.25 \mu \mathrm{m} ; 3080$ $\mathrm{cm}^{-1}$ ) observed in absorption toward molecular clouds. The longer wavelength of the 3.28 $\mu \mathrm{m}$ absorption suggests either that the aromatics are at a higher temperature, perhaps due to localized heating by sources in the Galactic Center, or that the composition, sizes, or ionization state of the aromatics observed in the diffuse ISM are different from the aromatics observed in molecular clouds.

The differences in observed width are also confusing. Laboratory studies [64] have shown that as individual PAHs are heated, their central wavelengths both shift to longer wavelengths and the width of the PAH band broadens. Figure 9, which has not been published elsewhere, illustrates the contrast between the short wavelength and broad width of the $3.25 \mu \mathrm{m}$ aromatic absorption in molecular clouds [19], and the longer wavelength and narrow width of the $3.28 \mu \mathrm{m}$ aromatic absorption towards the Galactic Center [66]. Both spectra have $\lambda / \Delta \lambda=1000$. Figure 9 also shows a spectrum of the $3.29 \mu \mathrm{m}$ IEF in emission 
in the reflection nebula NGC 7023, from the work in progress of myself, C. Moutou, A. Léger, L. Verstraete, M. Werner, M. Giard, and D. Rouan. This spectrum, with $\lambda / \Delta \lambda=$ 1000, demonstrates that the observed width of the $3.29 \mu \mathrm{m}$ IEF in emission (Sellgren et al. 2000, in preparation) is similar to or narrower than the $3.25 \mu \mathrm{m}$ feature in absorption [19], again in contradiction to the temperature model. This suggests that the observed wavelength shifts and widths are not due to temperature effects but rather to composition, size distribution, ionization, or other effects.

This mystery is underscored by recent observations of an absorption feature at 6.18 $\mu \mathrm{m}\left(1620 \mathrm{~cm}^{-1}\right)$, believed to be the aromatic $\mathrm{C}-\mathrm{C}$ stretch, toward five hot, mass-losing stars and two Galactic Center sources in ISO spectra [67]. A possible absorption feature at $6.24 \mu \mathrm{m}\left(1600 \mathrm{~cm}^{-1}\right)$ toward several protostars has also been tentatively identified as the $\mathrm{C}-\mathrm{C}$ stretching mode of aromatic hydrocarbons [20, 23]. If the $6.18 \mu \mathrm{m}$ absorption toward the sources observed by Schutte et al. [67] arises from aromatics in the diffuse ISM, the aromatics should again be cold with blue-shifted, narrow absorption features. The $6.24 \mu \mathrm{m}$ absorption feature observed by Keane et al. [23] toward protostars in molecular clouds, if due to aromatics, should also arise from cold aromatics with blue-shifted, narrow absorption features. The astronomical observations, however, do not fit this picture. The $6.18 \mu \mathrm{m}$ absorption feature observed through the diffuse ISM [67] is blue-shifted relative to the $6.22 \mu \mathrm{m}\left(1610 \mathrm{~cm}^{-1}\right)$ IEF in emission [68], as expected, but the $6.24 \mu \mathrm{m}$ absorption in molecular clouds is observed at a similar or slightly longer wavelength as the $6.22 \mu \mathrm{m} \mathrm{IEF}$ in emission. Furthermore, the $6.18 \mu \mathrm{m}$ absorption feature observed through the diffuse ISM is significantly narrower than the $6.24 \mu \mathrm{m}$ absorption feature observed towards molecular clouds, as shown in Figure 10 of Keane et al. [23]. Again this is strong evidence that both the central wavelengths and widths of aromatics observed in absorption and emission in the ISM are determined not by temperature or molecular physics of a single molecule, but rather by the composition mixture, size distribution, or mix of ionization states of aromatics observed along a particular line-of-sight.

\subsection{Need for Laboratory Work on PAHs in Water Ice Matrices}

One possible way to reconcile the temperature picture of the wavelength and width of aromatic absorption and emission with the astronomical observations is to consider whether aromatics in molecular clouds might be embedded in ice mantles on grains. Solid $\mathrm{H}_{2} \mathrm{O}$ ice is observed toward all sources in molecular clouds with detected 3.3 or $6.2 \mu \mathrm{m}$ aromatic absorption features. New astrochemical laboratory measurements at NASA's 
Ames Research Center are currently underway to measure the effect of a surrounding ice matrix on PAH absorption (M. Bernstein 2000, private communication). These results, once published, may clarify our understanding of aromatic absorption in molecular clouds. Measurements are needed for both neutral and ionized PAHs.

\section{Interstellar "Diamond-like" Carbon}

The broad $3.47 \mu \mathrm{m}\left(2880 \mathrm{~cm}^{-1}\right)$ absorption feature $\left(\mathrm{FWHM} \approx 0.1 \mu \mathrm{m}\right.$ or $\left.80 \mathrm{~cm}^{-1}\right)$ was first noted in ground-based spectra of four protostars by Allamandola et al. [69]. They suggested that the feature might be due to the $\mathrm{C}-\mathrm{H}$ stretch absorption of solo hydrogens attached to $s p^{3}$ bonded carbon clusters, the "diamond"-like form of carbon. The feature was present in every molecular cloud source looked at by Brooke et al. [21]. Interstellar microdiamonds, formed outside our solar system, have been identified in meteorites [70, 71] and so must therefore exist and survive in the diffuse ISM. Our observations [21, 22] of the $3.47 \mu \mathrm{m}$ absorption feature, however, reached the startling conclusion that the optical depth of the $3.47 \mu \mathrm{m}$ absorption feature is not correlated with the depth of silicate absorption, as might be expected for two refractory minerals such as diamonds and silicates (Figure 10). Instead, we found that the $3.47 \mu \mathrm{m}$ optical depth is strongly correlated with the depth of the $\mathrm{H}_{2} \mathrm{O}$ ice band, a very volatile ice that cannot exist outside molecular clouds (Figure 11).

\subsection{Need for Laboratory or Theoretical Frequencies for $\mathrm{C}-\mathrm{H}$ Bonds on the Surfaces of Microdiamonds}

Under the assumption that the $3.47 \mu \mathrm{m}$ feature is due to $\mathrm{C}-\mathrm{H}$ bonds, Brooke et al. [21] interpreted the correlation with $\mathrm{H}_{2} \mathrm{O}$ ice as indicating that both $\mathrm{C}-\mathrm{H}$ bonds and $\mathrm{H}_{2} \mathrm{O}$ ice form in step on molecular cloud dust by hydrogen addition reactions. However, we noted that other identifications of the $3.47 \mu \mathrm{m}$ feature are also possible. One fertile region for astrochemical research is to better establish the formation mechanism and absorption

spectrum of interstellar "diamond"-like carbon of interstellar size (1 nm; [70]) with surface hydrogen atoms. Predictions or measurements of the complete vibrational spectrum, including additional modes which could be searched for in astronomical spectra, would be particularly useful. It would also be useful to search for alternative identifications for the $3.47 \mu \mathrm{m}$ feature with simple molecules, composed of cosmically abundant elements (Fig. 
1), which would have sublimation temperatures close to that of $\mathrm{H}_{2} \mathrm{O}$ ice. Again, predictions or measurements of other vibrational modes that could be used to test the identification would be helpful.

\section{7. $\mathrm{C}_{60}^{+}$in the interstellar medium}

The fullerene $\mathrm{C}_{60}$ was first discovered in the laboratory by Kroto et al. [72]. They proposed that this novel form of aromatic carbon could potentially play an important role in the ISM. Theoretical studies of dust formation in carbon-rich stellar mass-loss [73 - 75] suggested that fullerenes could be formed in such carbon-rich environments and expelled into the ISM. Laboratory studies have also shown that one product of the photoerosion of hydrogenated amorphous carbon grains is fullerenes [76]. The existence of fullerenes such as $\mathrm{C}_{60}$ and $\mathrm{C}_{70}$ in our solar system, for instance in meteoritic samples, is hotly debated [77, 78].

Searches for $\mathrm{C}_{60}$ in the ISM, through its UV absorption band at $386 \mathrm{~nm}$, have placed stringent limits of $<0.01 \%$ of the cosmic carbon abundance in $\mathrm{C}_{60}[79,80]$. The dominant ionization state of $\mathrm{C}_{60}$ in the ISM, however, is predicted to be $\mathrm{C}_{60}^{+}[47,49,50]$. Foing \& Ehrenfreund [81, 82] detected diffuse interstellar bands (see [83] for a review of the diffuse interstellar bands) at 958 and $963 \mathrm{~nm}$, which they argued were due to $\mathrm{C}_{60}^{+}$, based on a comparison to laboratory data [84].

This inspired Moutou et al. [61] to use ISO data to search for the 7.1 and $7.5 \mu \mathrm{m}$

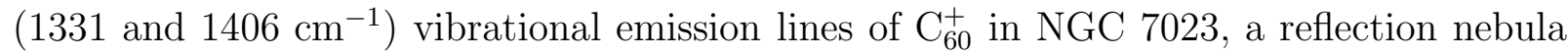
with strong IEF emission. Such a search requires high signal-to-noise and high spectral resolution $(\lambda / \Delta \lambda=1800)$, because these features, if present, would appear as weak bumps on the blue wing of the strong aromatic $7.7 \mu \mathrm{m}$ IEF. From our ISO spectra (Figure 8), we place an upper limit on $\mathrm{C}_{60}^{+}$in $\mathrm{NGC} 7023$ of $<0.3 \%$ of interstellar carbon [61].

The central star of NGC 7023, HD 200775, is known to have abnormally weak diffuse interstellar bands [85]. If the diffuse interstellar bands observed at 958 and $963 \mathrm{~nm}$ are due to $\mathrm{C}_{60}^{+}$, then the general weakness of the diffuse interstellar bands in NGC 7023 might naturally lead to a failure to detect $\mathrm{C}_{60}^{+}$in the mid-infrared toward this source. Astronomers can place more robust limits on the abundance of fullerenes in the ISM by searching for

the 7.1 and $7.5 \mu \mathrm{m} \mathrm{C}_{60}^{+}$lines towards a larger number of lines of sight, particularly those in which the diffuse interstellar bands are strong. 


\subsection{Laboratory and Theoretical Work Needed for Fullerenes}

Laboratory astrochemists can also contribute greatly to the search for interstellar fullerenes by further experiments. The assignment of the interstellar bands at 958 and 963 $\mathrm{nm}$ to $\mathrm{C}_{60}^{+}$was based on a comparison of gas-phase $\mathrm{C}_{60}^{+}$in the ISM with $\mathrm{C}_{60}^{+}$measured in inert-gas matrices, with the effects of matrix shifts on the wavelengths being unknown.

The 7.1 and $7.5 \mu \mathrm{m} \mathrm{C}_{60}^{+}$bands were also measured in inert-gas matrices, again introducing an unknown amount of matrix shift to the wavelengths. Laboratory measurements of all these bands in the gas phase would help immensely to determine whether detections of astronomical absorption features near the laboratory wavelengths of $\mathrm{C}_{60}^{+}$prove or disprove the existence of $\mathrm{C}_{60}^{+}$in the ISM. Finally, by symmetry, there should be two longer wavelength vibrational modes of $\mathrm{C}_{60}^{+}$which were not measured by Fulara et al. [84]. Laboratory wavelengths for these two additional modes would open up new avenues for astronomical observations of $\mathrm{C}_{60}^{+}$.

\section{Conclusions}

I have emphasized in this paper a number of puzzling astronomical observations of aromatics in the ISM, and related molecules, where understanding of these problems would greatly benefit from new experiments in laboratory astrochemistry and progress in chemical theory. The study of the ISM is an area where the interaction of astronomy, physics, and chemistry has proven particular fruitful in reaching new conclusions about the universe that surrounds us, and I strongly encourage astrochemistry groups to tackle these experimental and theoretical problems as part of this on-going interaction.

I would like to thank Tim Brooke for contributions to the text on the $3.47 \mu \mathrm{m}$ feature. I am grateful to Max Bernstein, Jean Chiar and Farid Salama for communicating data in advance of publication, and to Jacquie Keane for sharing a preprint before the refereeing process was complete. I also thank James Pizagno for speedy assistance with Figure 3.

\section{References}


[1] A.N. Cox, ed. 2000, Allen's Astrophysical Quantities, (4th ed.; New York: AIP/Springer), 29.

[2] D. Cesarsky, J. Lequeux, A. Abergel, M. Pérault, E. Palazzi, S. Madden, D. Tran, Astron. Astrophys. 315 (1996) L305.

[3] F.C. Gillett, W.J. Forrest, K.M. Merrill, Astrophys. J. 183 (1973) 87.

[4] M. Cohen, C.M. Anderson, A. Cowley, G.V. Coyne, W. Fawley, T.R. Gull, E.A. Harlan, G.H. Herbig, F. Holden, H.S. Hudson, R.O. Jakoubek, H.M. Johnson, K.M. Merrill, F.H. Schiffer, B.T. Soifer, B. Zuckerman, Astrophys. J. 196 (1975) 179.

[5] K.M. Merrill, B.T. Soifer, R.W. Russell, Astrophys. J. 200 (1975) L37.

[6] R.W. Russell, B.T. Soifer, S.P. Willner, Astrophys. J. 217 (1977) L153.

[7] P.F. Roche, D.K. Aitken, C.H. Smith, Mon. Not. Roy. Astron. Soc. 236 (1989) 485.

[8] P.F. Roche, D.K. Aitken, Mon. Not. Roy. Astron. Soc. 221 (1986) 63.

[9] M. Cohen, L. Allamandola, A.G.G.M. Tielens, J. Bregman, J.P. Simpson, F.C. Witteborn, D. Wooden, D. Rank, Astrophys. J. 302 (1986) 737.

[10] M. Cohen, A.G.G.M. Tielens, J. Bregman, F.C. Witteborn, D.M. Rank, L.J. Allamandola, D. Wooden, M. Jourdain de Muizon, Astrophys. J. 341 (1989) 246.

[11] J.L. Puget, A. Léger, Ann. Rev. Astron. Astrophys. 27 (1989) 161.

[12] L.J. Allamandola, A.G.G.M. Tielens, J.R. Barker, Astrophys. J. Suppl. 71 (1989) 733.

[13] K. Sellgren, A.T. Tokunaga, Y. Nakada, Astrophys. J. 349 (1990) 120.

[14] J.R. Graham, E. Serabyn, T.M. Herbst, K. Matthews, G. Neugebauer, B.T. Soifer, T.D. Wilson, S. Beckwith, Astron. J. 105 (1993) 250.

[15] F. Boulanger, W.T. Reach, A. Abergel, J.P. Bernard, C.J. Cesarsky, D. Cesarsky, F.X. Désert, E. Falgarone, J. Lequeux, L. Metcalfe, M. Pérault, J.L. Puget, D. Rouan, M. Sauvage, D. Tran, L. Vigroux, Astron. Astrophys. 315 (1996) L325.

[16] K. Mattila, D. Lemke, L.K. Haikala, R.J. Laureijs, A. Léger, K. Lehtinen, C. Leinert, P.G. Mezger, Astron. Astrophys. 315 (1996) L353.

[17] T. Onaka, I. Yamamura, T. Tanabe, T.L. Roellig, L. Yuen, Pub. Astron. Soc. Japan 48 (1996) L59.

[18] K. Sellgren, R.G. Smith, T.Y. Brooke, Astrophys. J. 433 (1994) 179. 
[19] K. Sellgren, T.Y. Brooke, R.G. Smith, T.R. Geballe, Astrophys. J. 449 (1995) L69.

[20] W.A. Schutte, A.G.G.M. Tielens, D.C.B. Whittet, A. Boogert, P. Ehrenfreund, Th. de Graauw, T. Prusti, E.F. van Dishoeck, P. Wesselius, Astron. Astrophys. 315 (1996) L333.

[21] T.Y. Brooke, K. Sellgren, R.G. Smith, Astrophys. J. 459 (1996) 209.

[22] T.Y. Brooke, K. Sellgren, T.R. Geballe, Astrophys. J. 517 (1999) 883.

[23] J.V. Keane, A.G.G.M. Tielens, A.C.A. Boogert, W.A. Schutte, D.C.B. Whittet, 2000, Astron. Astrophys., in press.

[24] W.W. Duley, D.A. Williams, Mon. Not. Roy. Astron. Soc. 196 (1981) 269.

[25] K. Sellgren, M.W. Werner, H.L. Dinerstein, Astrophys. J. 271 (1983) L13.

[26] K. Sellgren, Astrophys. J. 277 (1984) 623.

[27] K. Sellgren, M.W. Werner, H.L. Dinerstein, Astrophys. J. 400 (1992) 238.

[28] P.M. Harvey, H.A. Thronson, I. Gatley, Astrophys. J. 235 (1980) 894.

[29] S.E. Whitcomb, I. Gatley, R.H. Hildebrand, J. Keene, K. Sellgren, M.W. Werner, Astrophys. J. 246 (1981) 416.

[30] J.R. Platt, Astrophys. J. 123 (1956) 486.

[31] C.D. Andriesse, Astron. Astrophys. 66 (1978) 169.

[32] B.T. Draine, N. Anderson, Astrophys. J. 292 (1985) 494.

[33] J.L. Puget, A. Léger, F. Boulanger, Astron. Astrophys. 142 (1985) L19.

[34] K. Sellgren, L.J. Allamandola, J.D. Bregman, M.W. Werner, D.H. Wooden, Astrophys. J. 299 (1985) 416.

[35] A. Léger, J.L. Puget, Astron. Astrophys. 137 (1984) L5.

[36] L.J. Allamandola, A.G.G.M. Tielens, J.R. Barker, Astrophys. J. 290 (1985) L25.

[37] A. Sakata, S. Wada, T. Tanabe, T. Onaka, Astrophys. J. 287 (1984) L51.

[38] A. Sakata, S. Wada, T. Onaka, A.T. Tokunaga, Astrophys. J. 320 (1987) L63.

[39] A. Borghesi, E. Bussoletti, L. Colangeli, Astrophys. J. 314 (1987) 422.

[40] A. Blanco, E. Bussoletti, L. Colangeli, Astrophys. J. 334 (1988) 875. 
[41] W.W. Duley, Mon. Not. Roy. Astron. Soc. 234 (1988) 61P.

[42] R. Papoular, J. Conrad, M. Giuliano, J. Kister, G. Mille, Astron. Astrophys. 217 (1989) 204.

[43] K. Sellgren, L. Luan, M.W. Werner, Astrophys. J. 359 (1990) 384.

[44] K.I. Uchida, K. Sellgren, M. Werner, Astrophys. J. 493 (1998) L109.

[45] F. Salama, L.J. Allamandola, J. Chem. Phys. 94 (1991) 6964.

[46] K.I. Uchida, K. Sellgren, M.W. Werner, M.L. Houdashelt, Astrophys. J. 530 (2000) 817.

[47] E.L.O. Bakes, A.G.G.M. Tielens, Astrophys. J. 427 (1994) 822.

[48] E.L.O. Bakes, A.G.G.M. Tielens, Astrophys. J. 499 (1998) 258.

[49] F. Salama, E.L.O. Bakes, L.J. Allamandola, A.G.G.M. Tielens, Astrophys. J. 458 (1996) 621.

[50] E. Dartois, L. d'Hendecourt, Astron. Astrophys. 323 (1997) 534.

[51] D.J. de Frees, M.D. Miller, D. Talbi, F. Pauzat, Y. Ellinger, Astrophys. J. 408 (1993) 530.

[52] J. Szczepanski, M. Vala, Astrophys. J. 414 (1993) 646.

[53] D.M. Hudgins, S.A. Sandford, L.J. Allamandola, J. Phys. Chem. 98 (1994) 4243.

[54] F. Pauzat, D. Talbi, Y. Ellinger, Astron. Astrophys. 293 (1995) 263.

[55] F. Pauzat, D. Talbi, Y. Ellinger, Astron. Astrophys. 319 (1997) 318.

[56] S.R. Langhoff, J. Phys. Chem. 100 (1996) 2819.

[57] D.J. Cook, R.J. Saykally, Astrophys. J. 493 (1998) 793.

[58] L.J. Allamandola, D.M. Hudgins, S.A. Sandford, Astrophys. J. 511 (1999) L115.

[59] D.M. Hudgins, L.J. Allamandola, Astrophys. J. 516 (1999) L41.

[60] D. Cesarsky, J. Lequeux, C. Ryter, M. Gérin, Astron. Astrophys. 354 (2000) L87.

[61] C. Moutou, K. Sellgren, L. Verstraete, A. Léger, Astron. Astrophys. 347 (1999) 949.

[62] J.D. Bregman, L.J. Allamandola, A.G.G.M. Tielens, F.C. Witteborn, D.M. Rank, D. Wooden, 1986, in Summer School on Interstellar Processes: Abstracts of Contributed Papers, ed. D.J. Hollenbach, H.A. Thronson, 87. 
[63] J. Bregman, 1989, in Interstellar Dust, Internat. Astron. Union Symp. 135, ed. L.J. Allamandola, A.G.G.M. Tielens (Dordrecht: Kluwer Academic), 109.

[64] C. Joblin, P. Boissel, A. Léger, L. d'Hendecourt, D. Défourneau, Astron. Astrophys. 299 (1995) 835.

[65] Y.J. Pendleton, S.A. Sandford, L.J. Allamandola, A.G.G.M. Tielens, K. Sellgren, Astrophys. J. 437 (1994) 683.

[66] J.E. Chiar, A.G.G.M. Tielens, D.C.B. Whittet, W.A. Schutte, A.C.A. Boogert, D. Lutz, E.F. van Dishoeck, M.P. Bernstein, Astrophys. J. 537 (2000) 749.

[67] W.A. Schutte, K.A. van der Hucht, D.C.B. Whittet, A.C.A. Boogert, A.G.G.M. Tielens, P.W. Morris, J.M. Greenberg, P.M. Williams, E.F. van Dishoeck, J.E. Chiar, Th. de Graauw, Astron. Astrophys. 337 (1998) 261.

[68] D.A. Beintema, M.E. van den Ancker, F.J. Molster, L.F.B.M. Waters, A.G.G.M. Tielens, C. Waelkens, T. de Jong, Th. de Graauw, K. Justtanont, I. Yamamura, A. Heras, F. Lahuis, A. Salama, Astron. Astrophys. 315 (1996) L369.

[69] L.J. Allamandola, S.A. Sandford, A.G.G.M. Tielens, T.M. Herbst, Astrophys. J. 399 (1992) 134.

[70] R.S. Lewis, T. Ming, J.F. Wacker, E. Anders, E. Steel Nature 326 (1987) 160.

[71] E. Anders, E. Zinner, Meteoritics 28 (1993) 490.

[72] H.W. Kroto, J.R. Heath, S.C. O’Brien, R.F. Curl, R.E. Smalley, Nature 318 (1985) 162.

[73] H.W. Kroto, M. Jura, Astron. Astrophys. 263 (1992) 275.

[74] A. Goeres, E. Sedlmayr, Astron. Astrophys. 265 (1992) 216.

[75] R.P.A. Bettens, E. Herbst, Astrophys. J. 478 (1997) 585.

[76] A. Scott, W.W. Duley, G.P. Pinho, Astrophys. J. 489 (1997) L193.

[77] L. Becker, T.E. Bunch, Meteoritics 32 (1997) 479.

[78] D. Heymann, Astrophys. J. 489 (1997) L111.

[79] T.P. Snow, C.G. Seab, Astron. Astrophys. 213 (1989) 291.

[80] W.B. Somerville, J.G. Bellis, Mon. Not. Roy. Astron. Soc. 240 (1989) 41P.

[81] B.H. Foing, P. Ehrenfreund, Nature 369 (1994) 296.

[82] B.H. Foing, P. Ehrenfreund, Astron. Astrophys. 317 (1997) L59. 
[83] G.H. Herbig, Ann. Rev. Astron. Astrophys. 33 (1995) 19.

[84] J. Fulara, M. Jakobi, J.P. Maier, Chem. Phys. Letters 211 (1993) 227.

[85] R.D. Oudmaijer, G. Busfield, J.E. Drew, Mon. Not. Roy. Astron. Soc. 291 (1997) 797.

[86] J.E. Chiar, A.J. Adamson, D.C.B. Whittet, Astrophys. J. 472 (1996) 665. 


\section{Figure Captions}

Figure 1- The cosmic abundances of elements from Cox [1], presented as $\log _{10}$ of the elemental abundance relative to hydrogen versus the atomic number of the element. Only elements with abundances greater than $10^{-6}$ relative to hydrogen are illustrated.

Figure 2 - The ISOCAM + CVF spectrum of the reflection nebula NGC 7023 from Cesarsky et al. [2], plotted as relative flux density versus frequency in $\mathrm{cm}^{-1}$. The spectral resolution is $\nu / \Delta \nu=40$. Five of the six interstellar emission features (IEFs) can be seen, at $790,890,1160,1300$, and $1610 \mathrm{~cm}^{-1}(6.2,7.7,8.6,11.3$, and $12.7 \mu \mathrm{m})$.

Figure 3- IRAS broad-band photometric observations (filled squares) of $R$ (12/total), the ratio of the flux in the IRAS $12 \mu \mathrm{m}$ filter to the total infrared flux, plotted against $T_{\text {eff }}$ (star), the effective stellar temperature of the star which illuminates each reflection nebula, from Sellgren et al. [43]. Error bars are $\pm 1-\sigma$. Upper limits are 3- $\sigma$. The curves are the predicted values of $R(12 /$ total) from Pizagno et al. (2000, in preparation), derived by convolving the UV and visible absorption curves of neutral naphthalene (dashed curve), from Salama \& Allamandola [45], and ionized naphthalene (solid curve), from F. Salama (1999, private communication), with theoretical energy distributions of stars as a function of $T_{\text {eff }}($ star $)$.

Figure 4- Scaled ISOCAM + CVF spectra of three reflection nebulae, vdB 17 (NGC 1333; dotted line), vdB 59 (NGC 2068; thick solid line), and vdB 133 (thin solid line), plotted versus wavelength in $\mu \mathrm{m}$, from Uchida et al. [44, 46]. The spectral resolution is $\lambda / \Delta \lambda=40$. The stars which illuminate each reflection nebula respectively have $T_{\text {eff }}=$ $11,000 \mathrm{~K}$ for $\mathrm{vdB} 17,19,000 \mathrm{~K}$ for $\mathrm{vdB} 59$, and $6,800+12,000 \mathrm{~K}$ for $\mathrm{vdB} 133$ (which is illuminated by a binary star).

Figure 5- Ratio of the 5.5 - 9.75 $\mu \mathrm{m}$ flux to the 10.25 - $14.0 \mu \mathrm{m}$ flux versus the UV radiation field, $G_{0}$, from Uchida et al. [46]. All data are derived from ISOCAM + CVF spectra. Different nebular positions are illustrated for vdB 17 (filled diamonds; [46]), and vdB 59 (filled circles; [46]). Results at a single nebular position are shown for vdB 133 (open circle; [46]), NGC 7023 (cross; from Cesarsky et al. [2]), and $\rho$ Oph (open square; from Boulanger et al. [15]). Error bars are $\pm 1-\sigma$.

Figure 6- ISOCAM + CVF spectra of two reflection nebulae, plotted versus wavelength in $\mu \mathrm{m}$, from Uchida et al. [46]. The right panel is vdB 59 (NGC 2068), while 
the left panel is vdB 17 (NGC 1333). Spectra at different nebular positions are labeled by their values of the UV intensity, $G_{0}$. The spectral resolution is $\lambda / \Delta \lambda=40$. Note the broadening of the $7.7 \mu \mathrm{m}$ IEF at the lowest $G_{0}$ values in vdB 17 , which is unexplained by current models.

Figure 7- FWHM in $\mu \mathrm{m}$ of the $6.2,8.6$, and $7.7 \mu \mathrm{m}$ IEFs as a function of the UV radiation field, $G_{0}$, within vdB 17 , from Uchida et al. [46]. Data are derived from ISOCAM + CVF spectra. Error bars are $\pm 1-\sigma$. No correction has been made for the instrinsic spectral resolution of the instrument $(\lambda / \Delta \lambda=40)$.

Figure 8- Spectra at 7.0 - 8.7 $\mu \mathrm{m}$ of NGC 7023, plotted versus wavelength in $\mu \mathrm{m}$, observed with ISO's Short Wavelength Spectrometer (SWS) at $\lambda / \Delta \lambda=1800$ by Moutou et al. [61]. Solid vertical lines mark the laboratory positions of $\mathrm{C}_{60}^{+}$bands [84]. The data labeled "(up - down)/2" provide an estimate of the noise of the spectrum. The emission line in NGC 7023 at $8.02 \mu \mathrm{m}$ is the $0-0 \mathrm{~S}(4)$ line of molecular hydrogen. The SWS responsivity calibration is shown at the bottom of the plot, offset for clarity.

Figure 9- (top) Optical depth, plotted versus wavelength in $\mu \mathrm{m}$, of a possible aromatic absorption feature toward the protostar Mon R2/IRS-3, embedded in a molecular cloud, from ground-based spectra $(\lambda / \Delta \lambda=1000)$ obtained by Sellgren et al. [19] at the United Kingdom Infrared Telescope Facility. (middle) Optical depth, plotted versus wavelength in $\mu \mathrm{m}$, of a possible aromatic absorption feature toward the enigmatic Galactic Center source GCS3, which is obscured primarily by the diffuse interstellar medium, from Short Wavelength Spectrometer spectra $(\lambda / \Delta \lambda=1000)$ from ISO observed by Chiar et al. [66]. (bottom) Normalized intensity of the $3.3 \mu \mathrm{m}$ IEF toward the reflection nebula NGC 7023 (Sellgren et al. 2000, in preparation), from the Short Wavelength Spectrometer on ISO $(\lambda / \Delta \lambda=1000)$. The emission line at $3.234 \mu \mathrm{m}$ is the $1-0 \mathrm{O}(5)$ line of molecular hydrogen.

Figure 10 - The optical depth of the $3.47 \mu \mathrm{m}$ absorption band in molecular clouds, possibly due to "diamond"-like carbon, plotted versus the optical depth of $9.7 \mu \mathrm{m}$ silicate absorption, a refractory grain component. Data are from Brooke et al. [22] (filled circles), Sellgren et al. and Brooke et al. [18, 21] (open circles), and Chiar et al. [82] (crosses).

Figure 11 - The optical depth of the $3.47 \mu \mathrm{m}$ absorption band in molecular clouds, possibly due to "diamond"-like carbon, plotted versus the optical depth of $3.1 \mu \mathrm{m} \mathrm{H}_{2} \mathrm{O}$ ice absorption, a volatile grain component. Data are from Brooke et al. [22] (filled circles), Sellgren et al. and Brooke et al. [18, 21] (open circles), and Chiar et al. [82] (crosses). 


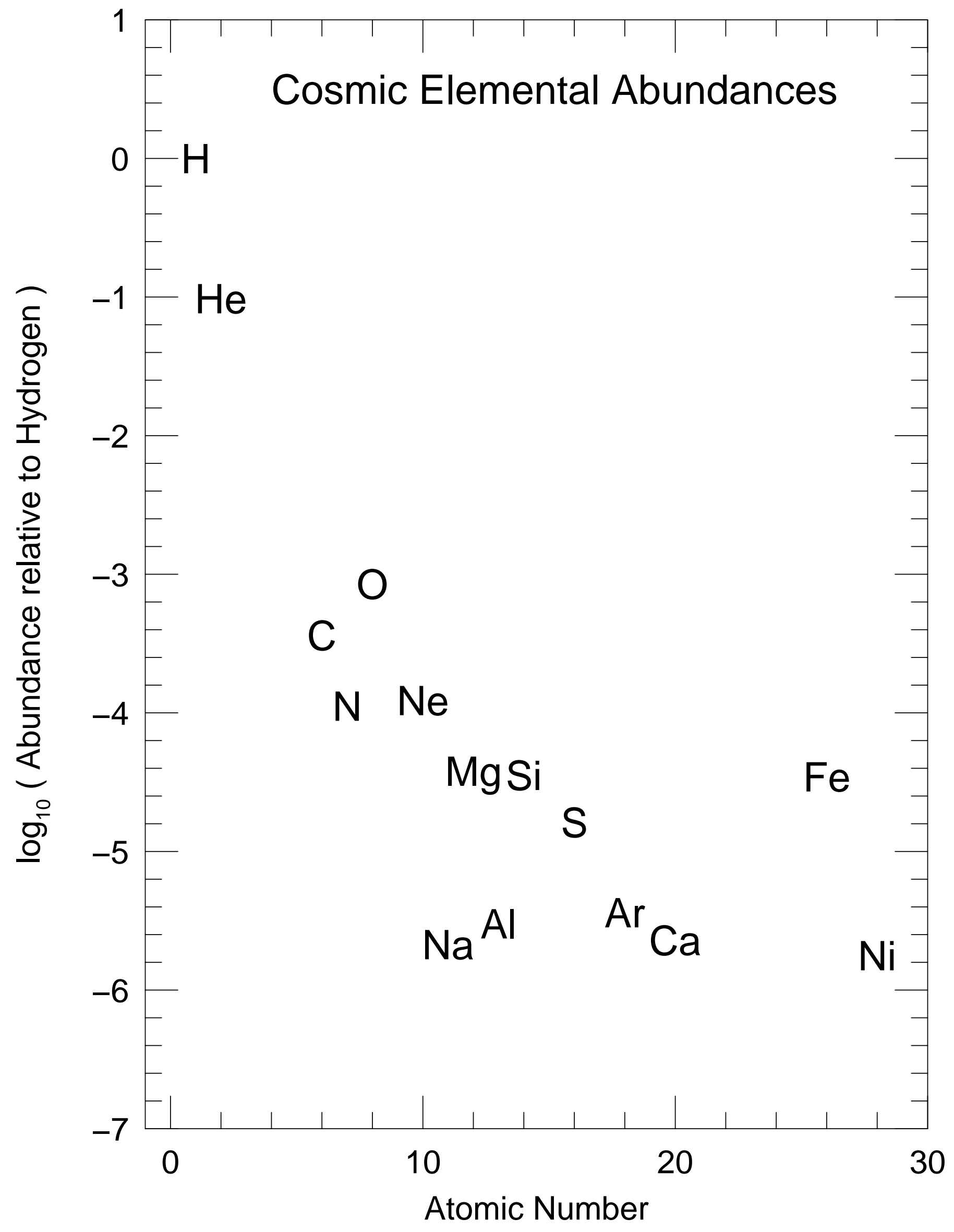




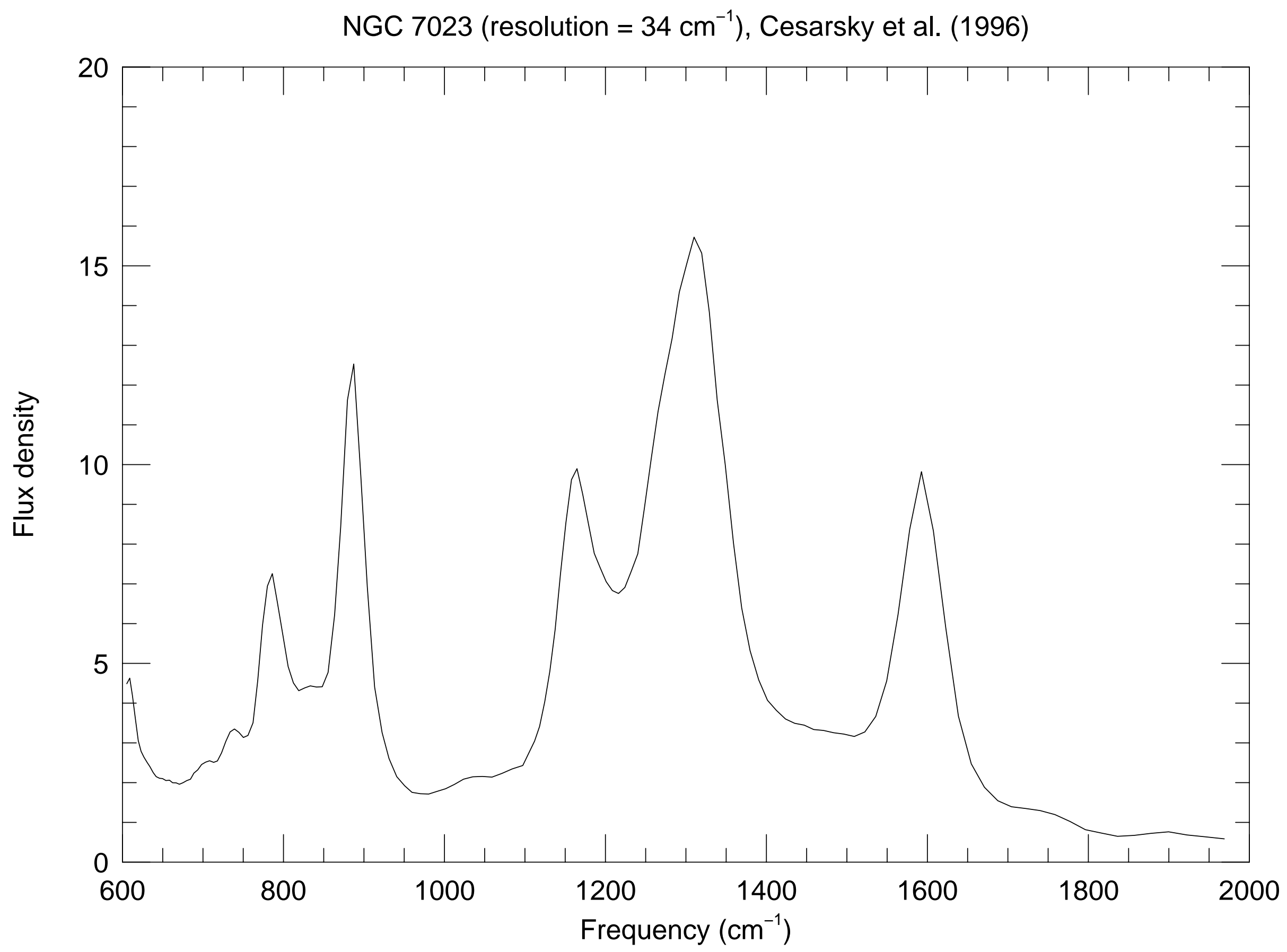




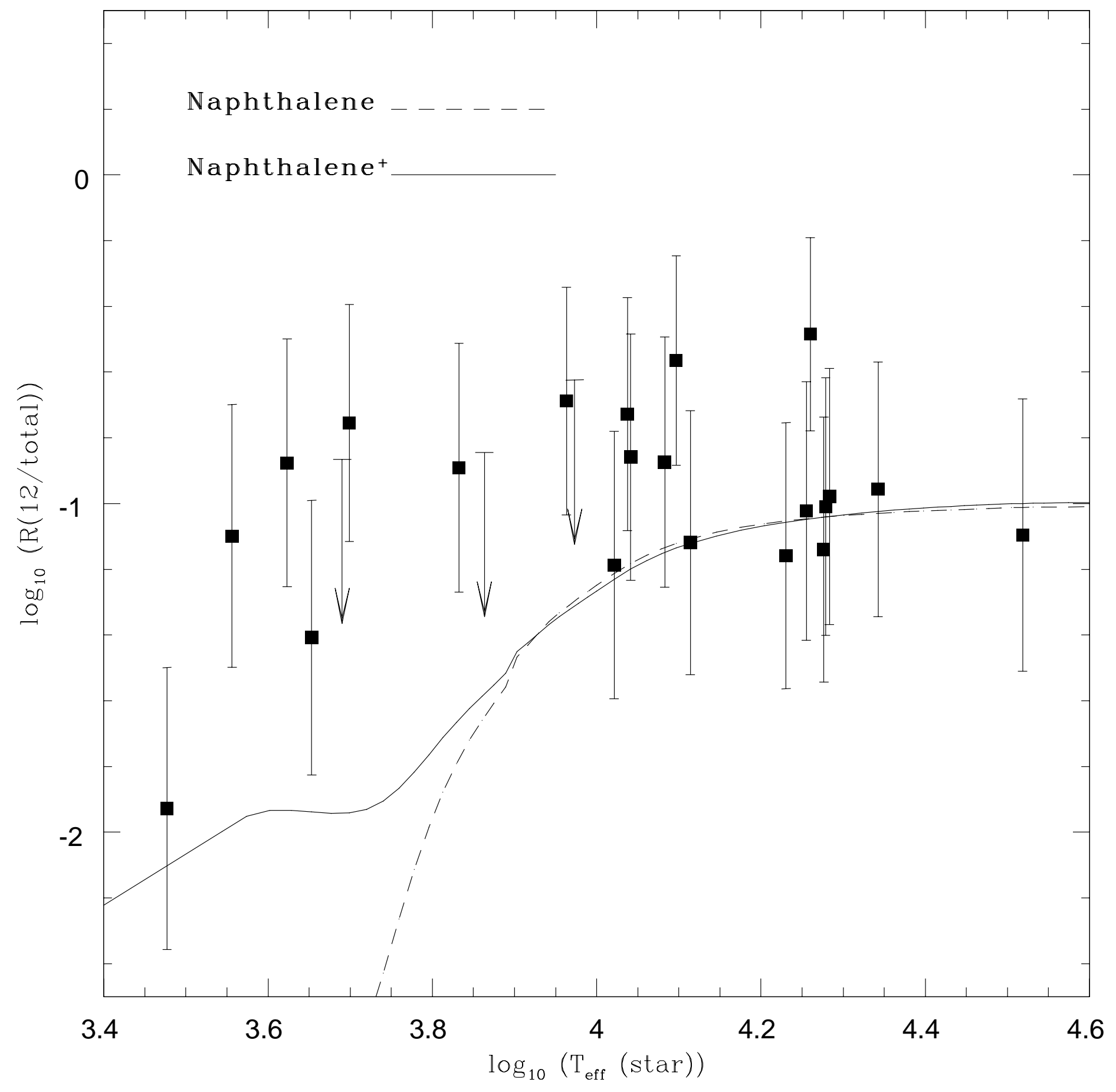




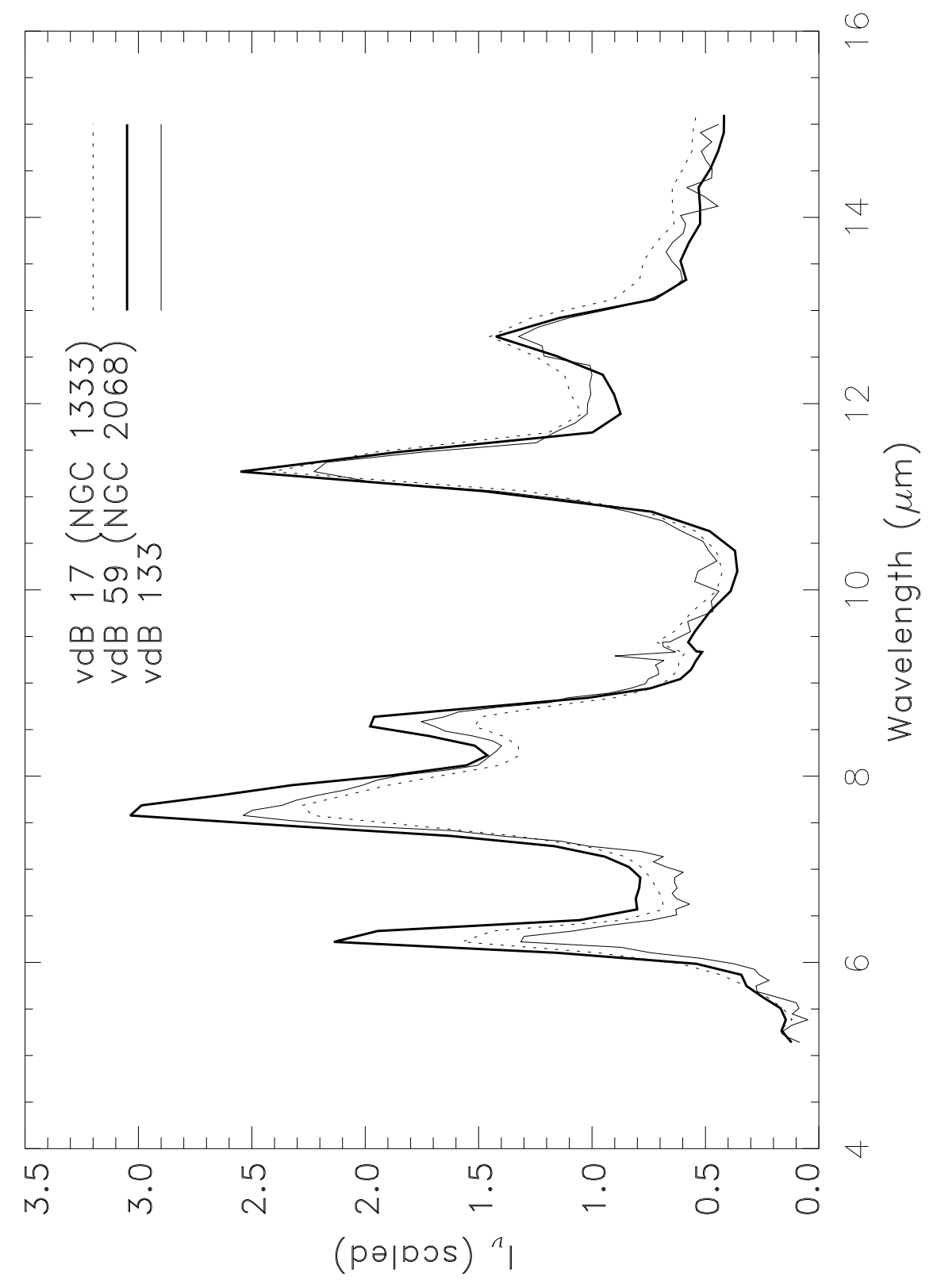




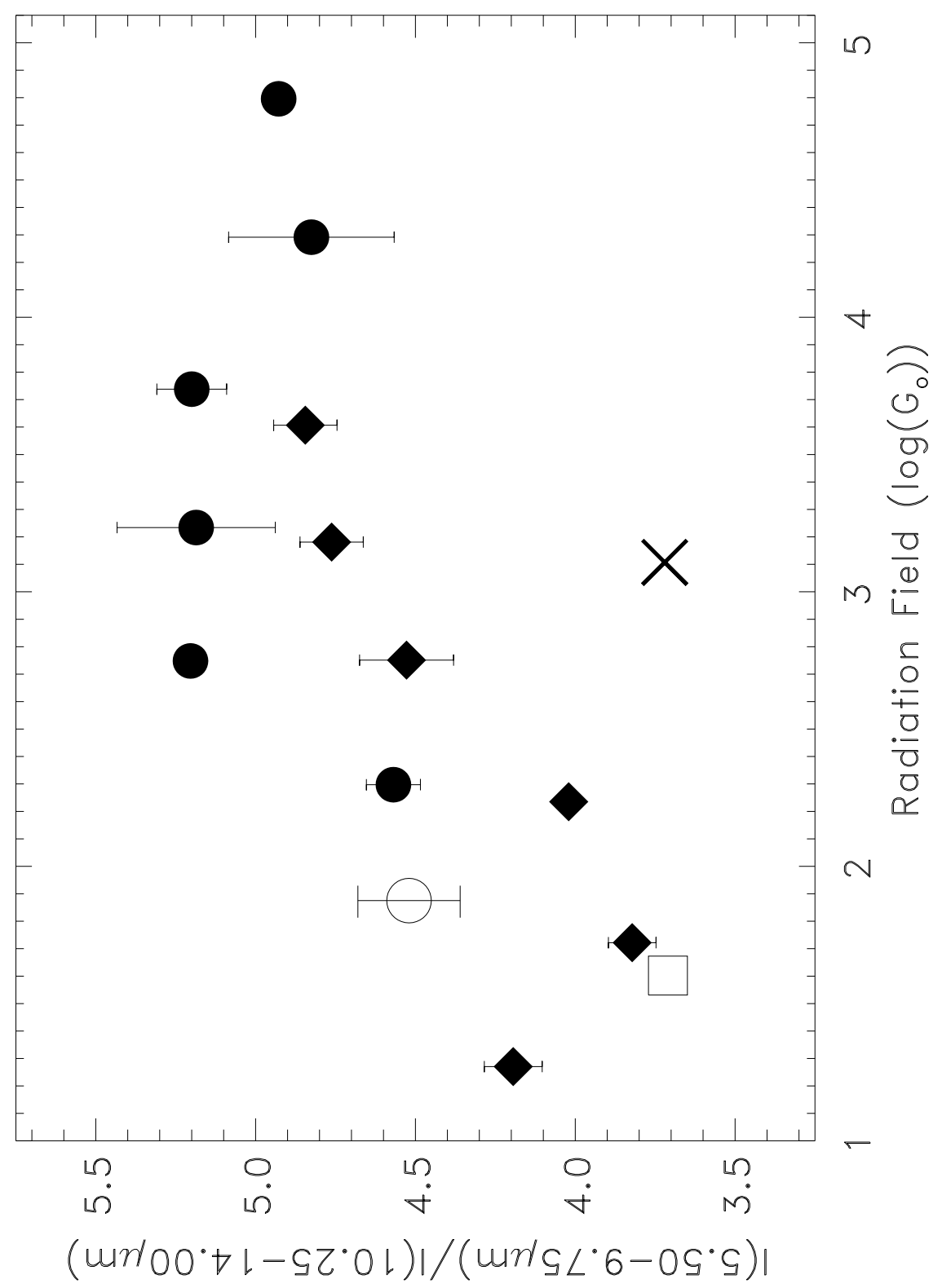



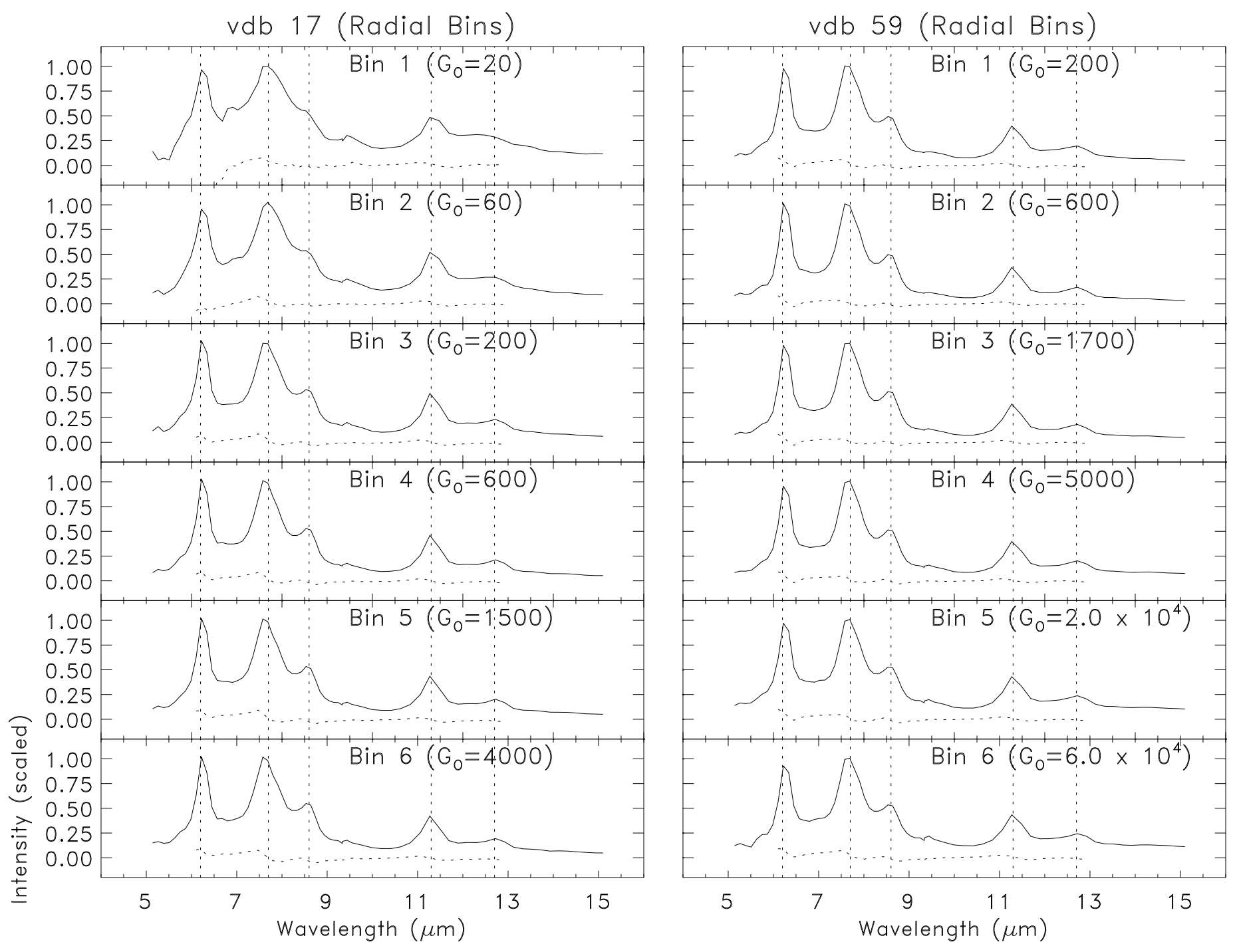


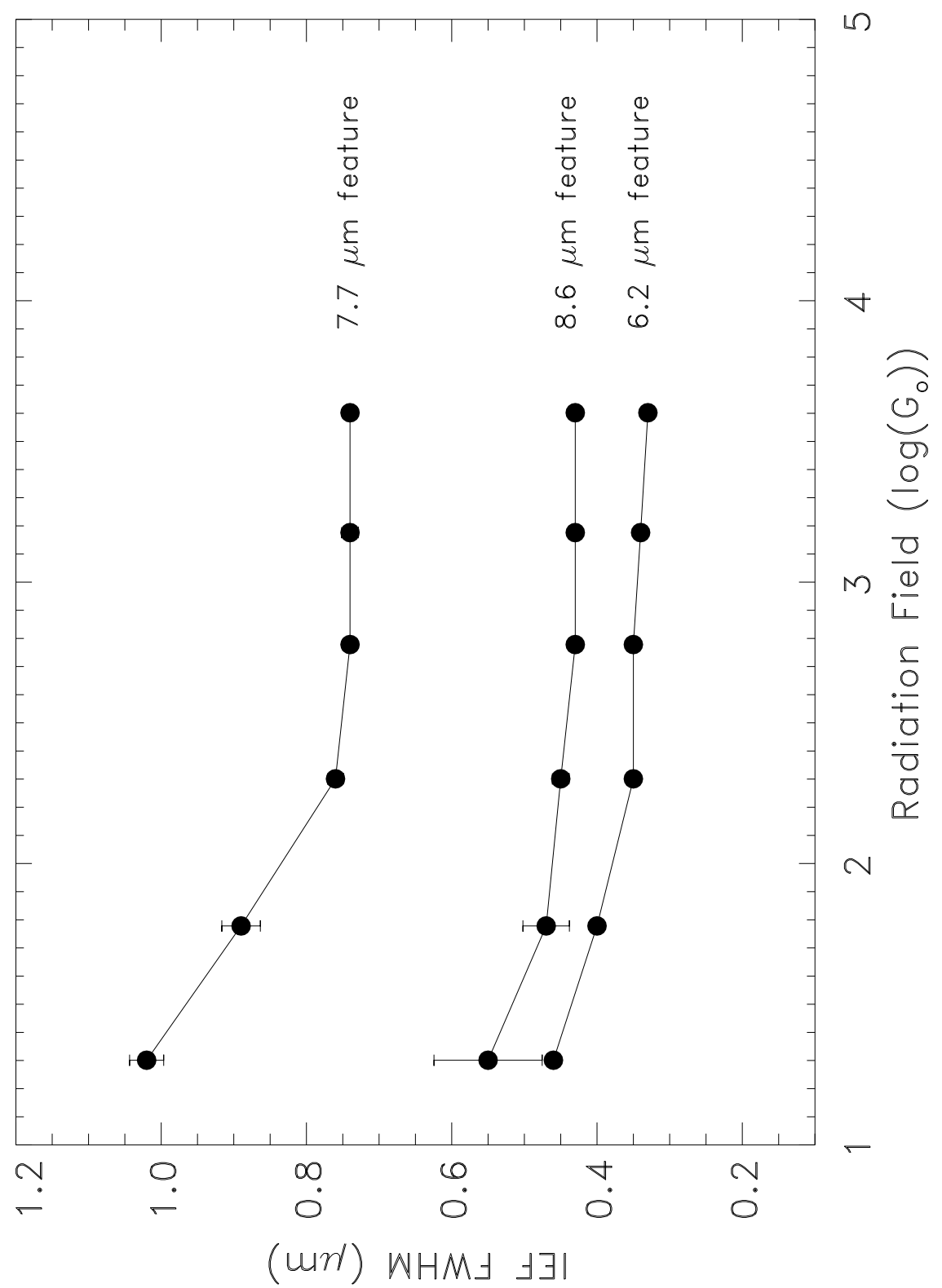




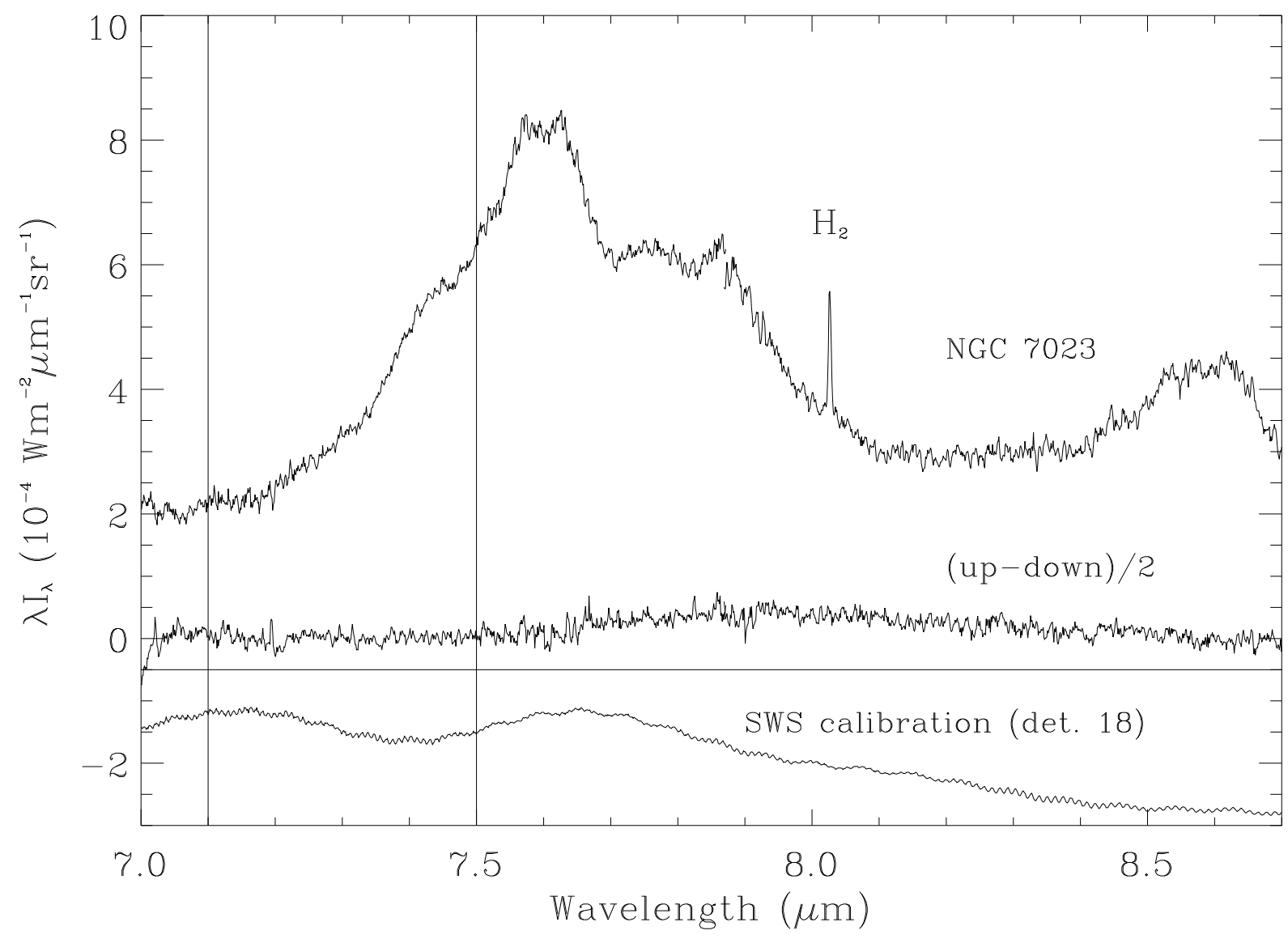




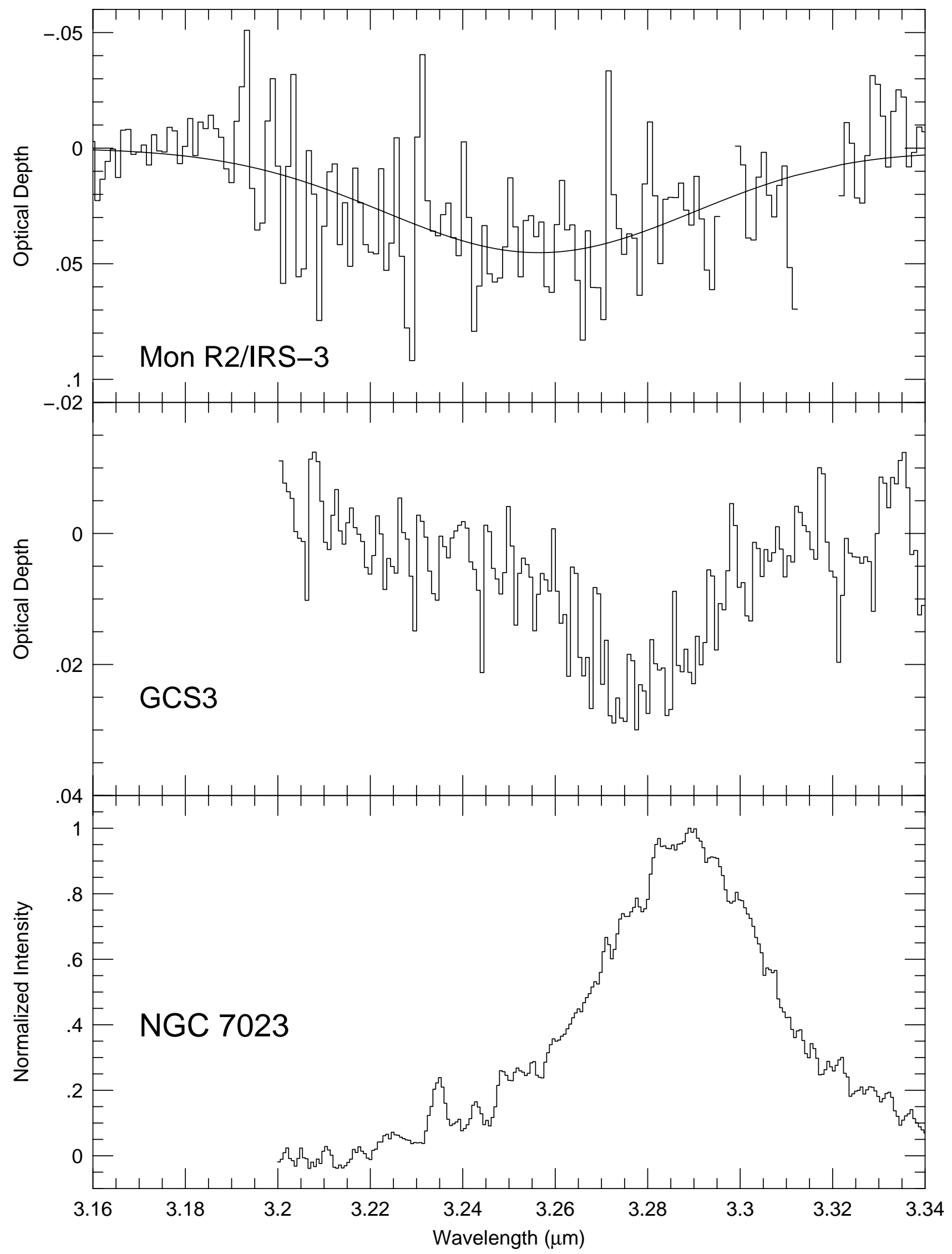




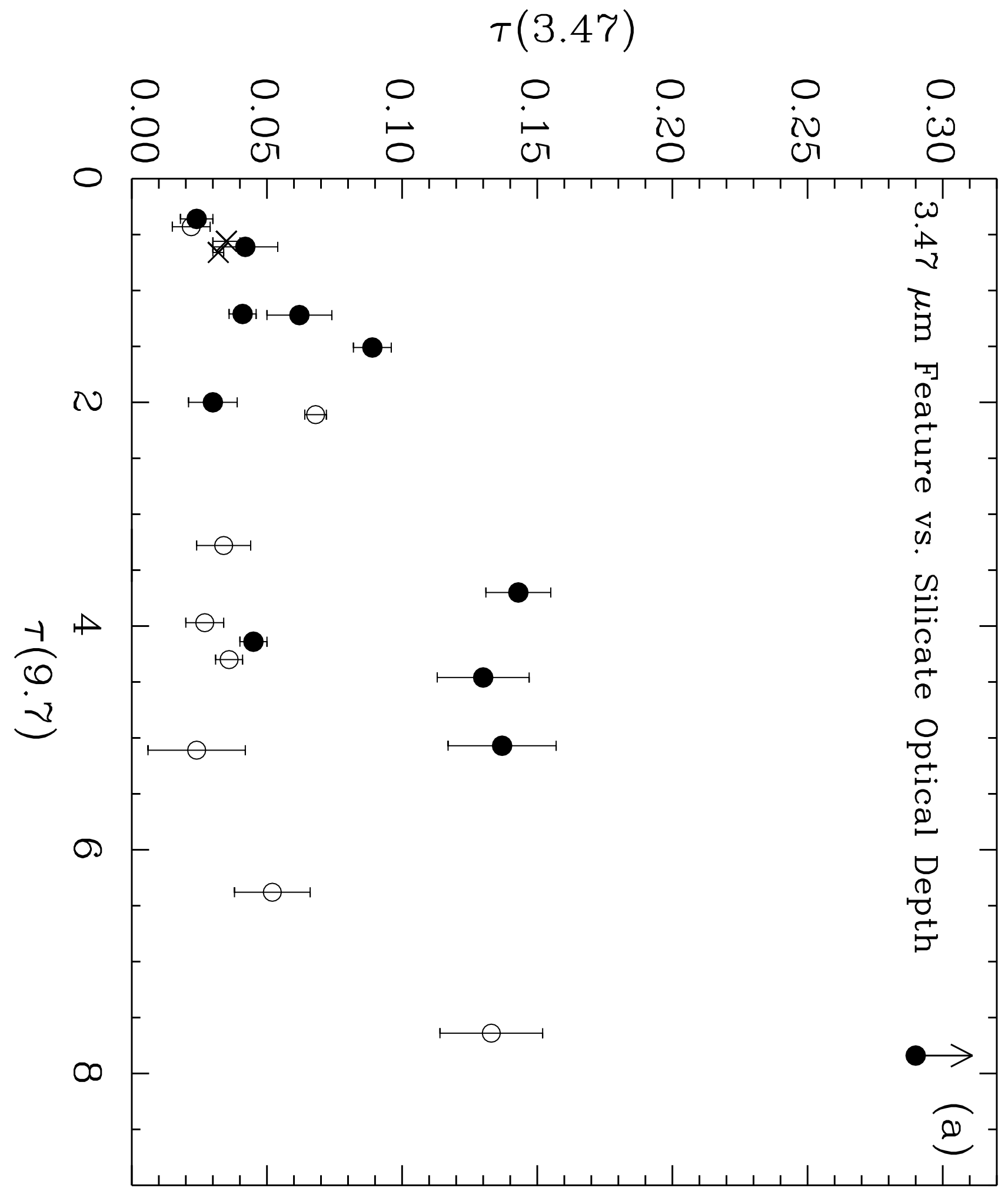




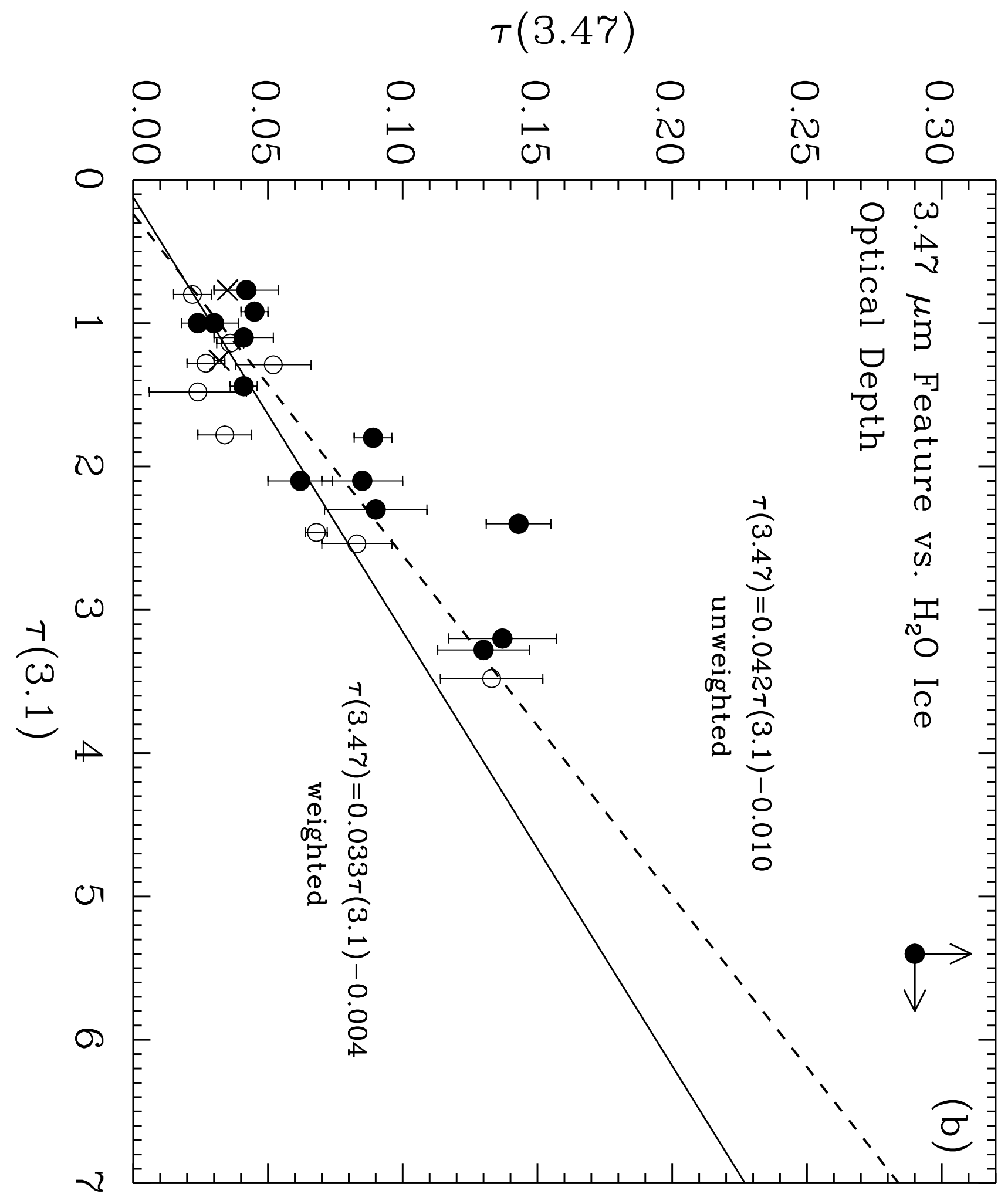

